\section{Primary malignant melanoma of the bronchus}

\author{
D J Farrell, A P Kashyap, T Ashcroft, \\ G N Morritt
}

\begin{abstract}
A rare case of primary malignant melanoma of the bronchus is described. Before considering this diagnosis, metastasis from an occult primary must be convincingly excluded and the tumour should conform to certain guidelines. This patient presented with a solitary lung tumour for which she underwent left lower lobectomy and continues to be tumour free 54 months after surgery. Two possible mechanisms of aetiology are suggested.

(Thorax 1996;51:223-224)
\end{abstract}

Keywords: bronchus, malignant melanoma.

Histopathology D J Farrell

T Ashcroft

Department of Cardiothoracic Surgery

A P Kashyap

G N Morritt

Freeman Hospital, High Heaton,

Newcastle upon Tyne, UK

Correspondence to:

Dr D J Farrell, Departmen

of Histopathology, Royal

Victoria Infirmary, Newcastle upon Tyne NE1 4LP, UK.

Received 16 January 1995 Returned to authors 18 April 1995

Revised version received 13 June 1995

Accepted for publication 16 June 1995

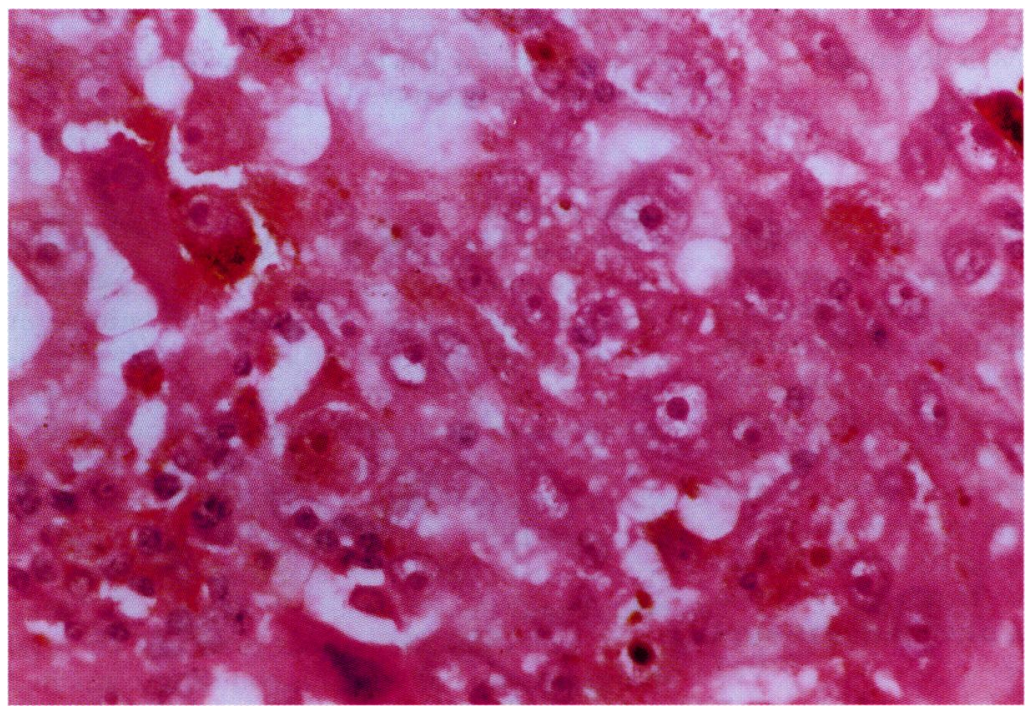

Microscopic section of tumour cells showing vesicular nuclei with large central nucleoli. Some also contain granular brown pigment. Stain: haematoxylin and eosin; original magnification $\times 840$ reduced to $59 \%$ in origination. in the left base and chest radiography demonstrated collapse and consolidation of the left lower lobe. Bronchoscopic examination revealed a polypoid endobronchial tumour at the origin of the left lower lobe bronchus which was not obviously pigmented. Biopsy samples showed it to be a malignant tumour and she subsequently underwent left lower lobectomy one week later. At operation the left lower lobe was collapsed and replaced by a tumour which was also loosely adherent to the diaphragm although not infiltrating it. The hilar, subcarinal, and subaortic lymph nodes were enlarged. Postoperatively the patient made a good recovery. Extensive examination of the skin, eyes, anorectal region, genitourinary tract, and oesophagus failed to reveal occult primary lesions.

\section{PATHOLOGICAL EXAMINATION}

The resected left lower lobe was inflated with formalin with some difficulty due to the obstructive tumour. Sectioning revealed a solitary irregular friable tumour $6 \mathrm{~cm}$ in greatest dimension within the superior segment, occluding the segmental bronchus and infiltrating the lobar bronchus. The cut surface was nodular and dark brown in colour with areas of haemorrhage and necrosis.

The bronchial resection margin was macroscopically free from tumour and the pleural surfaces were not involved.

Microscopic examination of the tumour showed that it was composed of large round cells and spindle cells arranged in closely packed sheets and islands. Most of the cells had vesicular nuclei with large central eosinophilic nucleoli and occasional mitotic figures were present. Large amounts of fine granular pigment were present within the tumour cells and macrophages (figure). Histochemical staining of this pigment showed a negative Prussian blue reaction. The pigment had a granular black appearance with the Masson-Fontana stain which was subsequently removed by bleaching indicating that this pigment was melanin. Immunohistochemical staining of the tumour cells showed that they expressed the S100 antigen but did not show expression of cytokeratins. The morphological appearances, histochemical, and immunohistochemical reactions of the tumour were those of a malignant melanoma. Examination of bronchial epithelium adjacent to the tumour showed areas of junctional activity in which nests of cells with large vesicular nuclei and eosinophilic nucleoli similar to the tumour cells were present above the basement membrane of intact bronchial epithelium. The melanocytic nature of these cells was confirmed by $\mathrm{S} 100$ staining which also revealed more of these cells showing upward migration through the bronchial epithelium and also lateral spread along the basement membrane. There was no lymph node involvement.

\section{Discussion}

True malignant melanoma of the bronchus is very rare and metastasis from the more common primary sources must be convincingly 
excluded before considering this diagnosis. It is difficult to prove that a malignant melanoma has its primary point of origin in internal organs because the possibility exists that it is a metastasis from a small primary tumour elsewhere, especially the skin or eye. The following criteria have therefore been devised by other authors ${ }^{23}$ which afford evidence to suggest primary occurrence in the lung:

(1) No previously resected pigmented skin lesion.

(2) No ocular tumour removed or evidence of a current or previous primary melanoma in any organ.

(3) Solitary tumour in the surgical specimen from the lung.

(4) Tumour morphology consistent with malignant melanoma involving the respiratory epithelium.

(5) Junctional change, with invasion of intact bronchial mucosa by malignant melanoma cells.

(6) Full necropsy demonstrating absence of primary malignant melanoma elsewhere.

Applying these criteria to previously reported cases in the literature greatly reduces the number of "probable" primary cases of malignant melanoma of the bronchus. ${ }^{23}$ In our case the patient presented with a solitary tumour showing all the morphological and staining characteristics of a malignant melanoma together with invasion of intact bronchial mucosa and convincing clinical evidence of lack of a primary tumour elsewhere.

Why should malignant melanomas develop in the bronchi when melanocytes are not present, apparently, in the normal respiratory tract? The most likely explanation is that these tumours arise from melanocytes which have migrated along with downgrowth of the primordial respiratory tract during fetal life from the primitive foregut, somewhere between the pharynx and the oesophagus, ${ }^{2}$ two sites at which malignant melanomas are known to occur. Alternatively, it has been speculated that tumour may arise from areas of squamous metaplasia ${ }^{4}$ in which certain of the epithelial cells have undergone differentiation towards melanocytes, a phenomenon which explains the occurrence of pigmented squamous and basal cell tumours in the skin. ${ }^{56}$ Melanocytes have also rarely been identified within the basal layer of the squamous epithelium in the larynx of a patient suffering from chronic laryngitis which suggests that a similar change could occur in irritated bronchi undergoing squamous metaplasia. $^{7}$

The long term survival of patients with primary malignant melanoma is unpredictable but is usually poor. Our patient remains well at 4.5 years following resection with no recurrence or metastases, which is further evidence of the likelihood that this represents a primary tumour. However, conclusive evidence may only be obtained after necroscopic examination.

1 Salm R. A primary malignant melanoma of bronchus. $f$ Pathol Bacteriol 1963;85:121-6.

2 Jensen OA, Egedorf J. Primary malignant melanoma of the lung. Scand F Respir Dis 1967;48:127-35.

3 Carstens PHB, Kuhns JG, Cyrus G. Primary malignant melanomas of the lung and adrenal. Hum Pathol 1984;15: melano-4.

4 Taboada CF, McMurray JO, Jordan RA, Seybold WD. Primary melanoma of the lung. Chest 1972;62:629-31.

5 Reid JD, Mehta VT. Melanoma of the lower respiratory tract. Cancer 1966;19:627-31.

6 Shivas AA, MacLennan WD. "Melanogenic metaplasia" of mucous glands. Br f Cancer 1963;17:411-4.

7 Busuttil A. Dendritic pigmented cells within human laryngeal mucosa. Arch Otolaryngol 1976;102:43-4.

Thorax 1996;51:224-226

Pulmonary Division, Cliniques

Universitaires Saint-

Luc, Brussels,

Belgium

Ph Collard

D O Rodenstein

Ch Francis

Ruhrlandklinik, Center for Thoracic Center for Thery and Pulmonary Medicine, Essen, Germany

L Freitag

Intensive Care Division, Cliniques

Universitaires Saint-

Luc, Brussels,

Belgium

M S Reynaert

Correspondence to:

$\mathrm{Dr} \mathrm{Ph}$ Collard, Cliniques

Universitaires Saint-Luc,

Service de Pneumologie,

Avenue Hippocrate 10

1200 Brussels, Belgium.

Received 20 October 1994

Returned to authors

16 January 1995

Revised version received

2 February 1995

Accepted for publication

21 February 1995

\section{Respiratory failure due to tracheobronchomalacia}

\author{
Ph Collard, L Freitag, M S Reynaert, \\ D O Rodenstein, Ch Francis
}

\begin{abstract}
A case is described of tracheobronchomegaly progressing to extensive tracheomalacia, complicated by episodic choking, recurrent pulmonary infections, and irreversible hypercapnic respiratory failure. A Y-shaped tracheobronchial stent was placed endoscopically to splint the trachea open, with excellent clinical and physiological improvement. New stent designs may provide long term palliation in selected cases of diffuse tracheal collapse or stenosis, and offer an alternative to surgical repair.
\end{abstract}

(Thorax 1996;51:224-226)

Keywords: tracheobronchomalacia, tracheobronchial stent, respiratory failure.
Tracheomalacia is a condition caused by weakness of the tracheal wall due to softening of the supporting cartilages. ${ }^{12}$ Initially it causes dynamic collapse during forced expiration and cough, which can be prevented by continuous positive airway pressure (CPAP). ${ }^{3}$ In the most severe cases the trachea becomes distorted and permanently narrowed.

\section{Case report}

A 52 year old man was diagnosed as having tracheobronchomegaly in 1982. In 1983 he received prolonged assisted ventilation for acute respiratory failure from bilateral bronchopneumonia. Fibreoptic bronchoscopy showed expiratory collapse of an enlarged trachea during spontaneous breathing, but the patency was maintained with a CPAP of $10 \mathrm{~cm} \mathrm{H}_{2} \mathrm{O}$. Between 1984 and 1986 he had three further episodes of acute respiratory failure necessitating mechanical ventilation. Between 1987 and 1990 the patient practised postural drainage twice daily and his condition remained stable.

In 1991 he developed difficulty in eliminating secretions despite postural drainage and he 